\title{
Finite frequency range iterative learning fault-tolerant control for discrete time-delay uncertain systems with actuator faults
}

\author{
Hongfeng Tao ${ }^{a}$ Wojciech Paszke ${ }^{b}$ Eric Rogers ${ }^{c}$ Huizhong Yang ${ }^{a}$ and Krzysztof \\ Galkowski b \\ a Key Laboratory of Advanced Process Control for Light Industry of Ministry of Education, \\ Jiangnan University, Wuxi 214122, PR China \\ ${ }^{b}$ Institute of Control and Computation Engineering, University of Zielona Góra, ul. \\ Podgórna 50, 65-246 Zielona Góra, Poland, Email: k.galkowski@issi.uz.zgora.pl \\ c School of Electronics and Computer Science, University of Southampton, Southampton \\ SO17 1BJ, UK Email: etar@ecs.soton.ac.uk
}

\section{ARTICLE HISTORY}

Compiled July 2, 2019

\begin{abstract}
The subject area considered is discrete linear time delay systems operating repetitively on a finite time interval with actuator faults, where the system resets at the end of each operation. Regulation of the dynamics is by iterative learning control and performance goals imposed over finite frequency intervals for the case of uncertainty in the dynamic model. To derive the results, the generalized Kalman-YakubovichPopov lemma is used. A simulation based case study is also given to demonstrate the applicability of the new results.
\end{abstract}

\section{KEYWORDS}

Iterative learning control, Finite frequency range, Fault tolerant control, Discrete time-delay systems, Repetitive processes

\section{Introduction}

Multidimensional systems propagate information in two or more directions and therefore variables are a function of two or more indeterminates. A particular example is processes with the distinguishing characteristic that the dynamics are defined over a finite duration and the mode of operation is based on executing over this duration followed by returning to the starting location and then repeating these two operations over and over again. The notation for variables in this paper is $y_{k}(p), 0 \leq p \leq \alpha-1$, where $y$ is the scalar of vector valued variable, the nonnegative integer $k$ is the execution or trial number and $\alpha$ is the number of samples over the finite duration, also known as the trial length.

Included within the class of multidimensional systems are those to which Iterative Learning Control (ILC) can be applied. This form of control forms the input on trial $k$ as the value used on the previous trial adjusted by a correction term that makes use of information generated on the previous trial, this information has already been generated and is available for use. A critical fact is that this allows the use of temporal information from the previous trial that would be noncausal in the non ILC setting. For 
example, at sample instant $p$ on the current trial information at sample $p+\lambda, \lambda>0$, on the previous trial can be used.

The first research on ILC appeared in the literature in the mid 1980's, for which Ahn, Chen, and Moore (2007); Bristow, Tharayil, and Alleyne (2006); Y. Wang, Gao, and Doyle III (2009) can be consulted as starting points for the literature. A critical result is that if an ILC law does not have a noncausal structure then it can be replaced by a feedback control law. A selection of applications where ILC laws have been used include additive manufacturing Lim, Hoelzle, and Barton (2016), robotic systems Hladowski et al. (2010); Longman (2000), multi-agent systems Meng and Moore (2016) and runto-run control (as a starting point in this last area see the relevant papers in Y. Wang et al. (2009)). These applications consist of finite duration tasks repeated over and over again with resetting to the starting location once completed, making ILC a very natural solution.

Alternative control designs may be possible for a particular application, e.g., iterative feedback tuning (IFT) Heertjes, Van der Velden, and Oomen (2016) where the controller parameters are obtained off-line without a detailed plant model. For a given application, the most suitable control design should be chosen and the focus on this paper is on further developing ILC design. Further development of the links between ILC and the subject area highlighted by Heertjes et al. (2016) is a topic for possible future research.

The design of an ILC law starts, as always, with performance specifications, where in the current case begins with the reference trajectory or vector, assumed, as in much of the literature, to be the same for all trials. For discrete-time dynamics, let $y_{k}(p)$, $u_{k}(p)$ denote the output and input vectors, respectively, on trial $k, 0 \leq p \leq \alpha-1$. Then if $y_{r}(p)$ denotes the reference vector, the error on trial $k$ is $e_{k}(p)=y_{r}(p)-y_{k}(p)$ and the basic task in ILC design is to construct a sequence of inputs that forces such that sequence $\lim _{k \rightarrow \infty} e_{k} \rightarrow 0$ and input sequence satisfies $\lim _{k \rightarrow \infty} u_{k} \rightarrow u_{\infty}$, where $u_{\infty}$ is termed the learned control vector. In applications, most often convergence to within a specified tolerance, as measured by an appropriate norm, will be the objective.

A common approach to ILC design for discrete dynamics is by a form of lifting. For single-input single-output (SISO) systems with a straightforward extension to the multiple-input multiple-output (MIMO) case the input and output on any trial can be represented by super-vectors formed by assembling the values at the sample instants into a column vector. Once the ILC law is applied, the propagation of the error from trial-to-trial can be represented by a linear difference equation and discrete linear systems theory can be used for trial-to-trial error convergence analysis and control law design.

Given the finite trial length, trial-to-trial error convergence can occur even if the system is unstable since such a system can only produce a bounded output over a finite time duration. Of course, there will be consequences for the transient dynamics along the trials in such a case and one solution is to design a feedback control law to stabilize the dynamics and then apply the ILC design to the controlled dynamics. A literature survey shows that this approach has been widely used in designs, some of which have been experimentally validated.

Given the finite trial length, convergence of the error sequence in $k$ can occur even if the system is unstable. In the lifting design setting, the only option is to design a pre-stabilizing feedback control and then apply ILC to the resulting control dynamics. This is a two step design and hence a possible conflict between stabilization and trialto-trial error convergence. An alternative is to do design in the 2D systems setting 
where one direction of information propagation is from trial-to-trial $(k)$ and the other along the trials $(\alpha)$.

Early work on the design of ILC laws using the systems theory for 2D linear systems includes Kurek and Zaremba (1993) where the Roesser state-space model was used. The Roesser model and alternatives describe 2D discrete linear systems recursive over the upper right quadrant of the $2 \mathrm{D}$ plane but ILC operates over a subset of this quadrant due to the finite trial length. Consequently, there is a more natural overlap with repetitive processes that are characterized by repeated sweeps through a set of dynamics defined over a finite duration, where once each sweep is complete the process resets to the starting location and the next one can begin.

In a repetitive process the output on the previous sweep, termed a trial with the associated trial length in this paper, acts as a forcing function on the next trial and the result can be oscillations that increase in amplitude from trial-to-trial. Moreover, standard control theory cannot regulate these processes. This has required the development of a stability theory for repetitive processes where it is required that a bounded initial trial profile produces a bounded sequence of trial profiles. For these processes, boundedness is defined by the norm assumed on the underlying function space. Consult Rogers, Gałkowski, and Owens (2007) for the details of this theory and how it can be extended to allow control law design for stability and performance. See also Hladowski et al. (2010); Paszke, Rogers, Galkowski, and Cai (2013) for the use of this setting to design an ILC law experimentally verified on a gantry robot test facility.

Time-delays are commonly encountered in many control applications, e.g., a physical variable, such as temperature, cannot be measured instantaneously. The resulting time delay is often the source of instability and poor performance of a control system, see, e.g., Gu, Kharitonov, and Chen (2003). Chemical processes are a general area where examples of dynamics that must be modeled as a system with delay(s) arise. This general area has been the subject of previous ILC research using the 2D systems setting, e.g., by treating them as batch processes with a finite time for each batch Y. Wang et al. (2009).

Industrial control systems often operate under challenging conditions and are consequently exposed to faults that, in turn, could cause a loss of stability or performance degradation (such as fatigue and components abrasion). Implementation of an ILC law imposes repetitive tasks on the actuators used, which increases the likelihood of a fault. Fault tolerance is therefore an issue for ILC schemes and this subject area has seen previous research, e.g. L. Wang, Mo, Zhou, et al. (2012) where a delay dependent iterative learning fault-tolerant control scheme is considered. However, designs such as this one enforce frequency attenuation over all of the frequency spectrum and this is often very restrictive/not possible to achieve.

Design specifications for many application are imposed over finite frequency ranges of interest. Previous work Paszke, Rogers, and Gałkowski (2016) in the fault free case has shown that such a design is possible by making use of the GKYP lemma, with follow through to experimental verification. This paper develops new results for ILC design for linear discrete systems with actuator faults. The new developments: (a) finite frequency range ILC law design is extended to the fault tolerant control problem for discrete linear time-delay systems with actuator faults, (b) monotonic trial-to-trial error convergence conditions for the controlled ILC dynamics, (c) robust control against structured uncertainty. Results for both state and output feedback based designs are developed. The focus in this paper is on the development of control law design algorithms, with, as an essential step before experimental validation/application to a 
physical example, a simulation based case study using the model of a physical process.

\section{Introduction}

Multidimensional systems propagate information in two or more directions and therefore variables are a function of two or more indeterminates. A particular example is processes with the distinguishing characteristic that the dynamics are defined over a finite duration and the mode of operation is based on executing over this duration followed by returning to the starting location and then repeating these two operations over and over again. The notation for variables in this paper is $y_{k}(p), 0 \leq p \leq \alpha-1$, where $y$ is the scalar of vector valued variable, the nonnegative integer $k$ is the execution or trial number and $\alpha$ is the number of samples over the finite duration, also known as the trial length.

Included within the class of multidimensional systems are those to which Iterative Learning Control (ILC) can be applied. This form of control forms the input on trial $k$ as the value used on the previous trial adjusted by a correction term that makes use of information generated on the previous trial, this information has already been generated and is available for use. A critical fact is that this allows the use of temporal information from the previous trial that would be noncausal in the non ILC setting. For example, at sample instant $p$ on the current trial information at sample $p+\lambda, \lambda>0$, on the previous trial can be used.

The first research on ILC appeared in the literature in the mid 1980's, for which Ahn et al. (2007); Bristow et al. (2006); Y. Wang et al. (2009) can be consulted as starting points for the literature. A critical result is that if an ILC law does not have a noncausal structure then it can be replaced by a feedback control law. A selection of applications where ILC laws have been used include additive manufacturing Lim et al. (2016), robotic systems Hladowski et al. (2010); Longman (2000), multi-agent systems Meng and Moore (2016) and run-to-run control (as a starting point in this last area see the relevant papers in Y. Wang et al. (2009)). These applications consist of finite duration tasks repeated over and over again with resetting to the starting location once completed, making ILC a very natural solution.

Alternative control designs may be possible for a particular application, e.g., iterative feedback tuning (IFT) Heertjes et al. (2016) where the controller parameters are obtained off-line without a detailed plant model. For a given application, the most suitable control design should be chosen and the focus on this paper is on further developing ILC design. Further development of the links between ILC and the subject area highlighted by Heertjes et al. (2016) is a topic for possible future research.

The design of an ILC law starts, as always, with performance specifications, where in the current case begins with the reference trajectory or vector, assumed, as in much of the literature, to be the same for all trials. For discrete-time dynamics, let $y_{k}(p)$, $u_{k}(p)$ denote the output and input vectors, respectively, on trial $k, 0 \leq p \leq \alpha-1$. Then if $y_{r}(p)$ denotes the reference vector, the error on trial $k$ is $e_{k}(p)=y_{r}(p)-y_{k}(p)$ and the basic task in ILC design is to construct a sequence of inputs that forces such that sequence $\lim _{k \rightarrow \infty} e_{k} \rightarrow 0$ and input sequence satisfies $\lim _{k \rightarrow \infty} u_{k} \rightarrow u_{\infty}$, where $u_{\infty}$ is termed the learned control vector. In applications, most often convergence to within a specified tolerance, as measured by an appropriate norm, will be the objective.

A common approach to ILC design for discrete dynamics is by a form of lifting. For single-input single-output (SISO) systems with a straightforward extension to the 
multiple-input multiple-output (MIMO) case the input and output on any trial can be represented by super-vectors formed by assembling the values at the sample instants into a column vector. Once the ILC law is applied, the propagation of the error from trial-to-trial can be represented by a linear difference equation and discrete linear systems theory can be used for trial-to-trial error convergence analysis and control law design.

Given the finite trial length, trial-to-trial error convergence can occur even if the system is unstable since such a system can only produce a bounded output over a finite time duration. Of course, there will be consequences for the transient dynamics along the trials in such a case and one solution is to design a feedback control law to stabilize the dynamics and then apply the ILC design to the controlled dynamics. A literature survey shows that this approach has been widely used in designs, some of which have been experimentally validated.

Given the finite trial length, convergence of the error sequence in $k$ can occur even if the system is unstable. In the lifting design setting, the only option is to design a pre-stabilizing feedback control and then apply ILC to the resulting control dynamics. This is a two step design and hence a possible conflict between stabilization and trialto-trial error convergence. An alternative is to do design in the 2D systems setting where one direction of information propagation is from trial-to-trial $(k)$ and the other along the trials $(\alpha)$.

Early work on the design of ILC laws using the systems theory for 2D linear systems includes Kurek and Zaremba (1993) where the Roesser state-space model was used. The Roesser model and alternatives describe 2D discrete linear systems recursive over the upper right quadrant of the 2D plane but ILC operates over a subset of this quadrant due to the finite trial length. Consequently, there is a more natural overlap with repetitive processes that are characterized by repeated sweeps through a set of dynamics defined over a finite duration, where once each sweep is complete the process resets to the starting location and the next one can begin.

In a repetitive process the output on the previous sweep, termed a trial with the associated trial length in this paper, acts as a forcing function on the next trial and the result can be oscillations that increase in amplitude from trial-to-trial. Moreover, standard control theory cannot regulate these processes. This has required the development of a stability theory for repetitive processes where it is required that a bounded initial trial profile produces a bounded sequence of trial profiles. For these processes, boundedness is defined by the norm assumed on the underlying function space. Consult Rogers et al. (2007) for the details of this theory and how it can be extended to allow control law design for stability and performance. See also Hladowski et al. (2010); Paszke et al. (2013) for the use of this setting to design an ILC law experimentally verified on a gantry robot test facility.

Time-delays are commonly encountered in many control applications, e.g., a physical variable, such as temperature, cannot be measured instantaneously. The resulting time delay is often the source of instability and poor performance of a control system, see, e.g., Gu et al. (2003). Chemical processes are a general area where examples of dynamics that must be modeled as a system with delay(s) arise. This general area has been the subject of previous ILC research using the 2D systems setting, e.g., by treating them as batch processes with a finite time for each batch L. Wang, Mo, Zhou, et al. (2013).

Industrial control systems often operate under challenging conditions and are consequently exposed to faults that, in turn, could cause a loss of stability or performance degradation (such as fatigue and components abrasion). Implementation of an ILC 
law imposes repetitive tasks on the actuators used, which increases the likelihood of a fault. Fault tolerance is therefore an issue for ILC schemes and this subject area has seen previous research, e.g. L. Wang et al. (2012) where a delay dependent iterative learning fault-tolerant control scheme is considered. However, designs such as this one enforce frequency attenuation over all of the frequency spectrum and this is often very restrictive/not possible to achieve.

Design specifications for many application are imposed over finite frequency ranges of interest. Previous work Paszke et al. (2016) in the fault free case has shown that such a design is possible by making use of the GKYP lemma, with follow through to experimental verification. This paper develops new results for ILC design for linear discrete systems with actuator faults. The new developments: (a) finite frequency range ILC law design is extended to the fault tolerant control problem for discrete linear time-delay systems with actuator faults, (b) monotonic trial-to-trial error convergence conditions for the controlled ILC dynamics, (c) robust control against structured uncertainty. Results for both state and output feedback based designs are developed. The focus in this paper is on the development of control law design algorithms, with, as an essential step before experimental validation/application to a physical example, a simulation based case study using the model of a physical process.

The remainder of this paper is organized as follows. Section 3 describes a class of discrete linear time delay systems in the ILC setting by a state-space model with actuator faults. Section 4 formulates the ILC design problem in the repetitive process setting for analysis. Then the monotonic convergence and stability of the controlled dynamic are analyzed in Section 5. Section 6 gives the fault tolerant ILC law design subject to the corresponding LMI constraints. Section 7 verifies the effectiveness of this method by a simulation based case. Finally, conclusions and areas for future research are given in Section 8.

Throughout this paper, the following notation is used. The null and identity matrices with the required dimensions are denoted by 0 and $I$, respectively, $X \prec Y$ (respectively $X \succ Y)$ is used to represent the negative definite (respectively, positive definite) matrix $X-Y$. The notation $(\star)$ denotes transposed elements in a symmetric matrix and $\rho(\cdot)$ denotes the spectral radius of its matrix argument, i.e., if $\lambda_{i}, 1 \leq i \leq q$, denote the eigenvalues of a $q \times q$ matrix, say $M, \rho(M)=\max _{1 \leq i \leq q}\left|\lambda_{i}\right|$. The symbol $\operatorname{diag}\left\{X_{1}, X_{2}, \ldots, X_{n}\right\}$ denotes a block diagonal matrix with diagonal blocks $X_{1}, X_{2}$, $\cdots, X_{n}$ and $\operatorname{sym}(\Lambda)=\Lambda+\Lambda^{T}$. Finally, $\otimes$ denotes the Kronecker matrix product and the superscript $*$ denotes the complex conjugate transpose of a matrix.

The new results in this paper make use of the following results, where the first is the variable elimination lemma and the second is the projection lemma.

Lemma 2.1. Iwasaki and Hara (2005) Given matrices $X, Y, \Phi=\Phi^{T}, \delta(p)$ of compatible dimensions, then

$$
\Phi+\operatorname{sym}\{X \delta(p) Y\} \prec 0,
$$

for all $\delta(p)$ satisfying $\delta^{T}(p) \delta(p) \preceq I$ if, and only if, there exists $\varepsilon>0$ such that

$$
\Phi+\varepsilon X X^{T}+\varepsilon^{-1} Y^{T} Y \prec 0 .
$$

Lemma 2.2. Gahinet and Apkarian (1994) Let $\Upsilon=\Upsilon^{T}, \Lambda$ and $\Sigma$ be given matrices of compatible dimensions. Then the following statements are equivalent 
- there exists an unstructured matrix $W$ that satisfies

$$
\Upsilon+\operatorname{sym}\left\{\Lambda^{T} W \Sigma\right\} \prec 0,
$$

- for arbitrary matrices $\Lambda_{\perp}$ and $\Sigma_{\perp}$ whose columns form, respectively, a basis for the null spaces of $\Lambda$ and $\Sigma$ the following inequalities hold

$$
\Lambda_{\perp}^{T} \Upsilon \Lambda_{\perp} \prec 0, \text { and } \Sigma_{\perp}^{T} \Upsilon \Sigma_{\perp} \prec 0 .
$$

\section{System description}

Consider the class of uncertain discrete linear systems with a single delay and dynamics described in the ILC setting by the following state-space model over $0 \leq p \leq \alpha-1, k \geq$ 0 as

$$
\begin{aligned}
x_{k}(p+1)= & (A+\Delta A(p)) x_{k}(p)+\left(A_{d}+\Delta A_{d}(p)\right) x_{k}(p-d) \\
& +(B+\Delta B(p)) u_{k}(p), \\
y_{k}(p)= & C x_{k}(p),
\end{aligned}
$$

where the subscript $k$ denotes the trial number and $\alpha$ the number of samples over the finite trial length. Also $x_{k}(p) \in \Re^{n}, y_{k}(p) \in \Re^{l}$ and $u_{k}(p) \in \Re^{m}$ represent the system state, output and input vectors at sample instant $p$ and trial number $k$ respectively; $d>0$ is the unknown time-delay constant satisfying $0<d \leq \bar{d}$, and $\bar{d}$ is a known upper bound. The time-varying uncertainties associated with the dynamics are modeled as additive perturbations and assumed to be of norm-bounded form and described by

$$
\Delta A(p)=E_{1} \delta(p) F_{1}, \Delta A_{d}(p)=E_{1} \delta(p) F_{d}, \Delta B(p)=E_{2} \delta(p) F_{2},
$$

where $E_{1}, E_{2}, F_{1}, F_{2}$ and $F_{d}$ are known and of compatible dimensions, and $\delta(p)$ is an unknown and time-varying perturbation satisfying $\delta^{T}(p) \delta(p) \preceq I, \forall p \in[0, \alpha-1]$. Also, $\delta(p)$ is assumed to be independent of the trial number $k$ and therefore the allowable uncertainties can vary along each trial but are assumed to be constant from trial-totrial. No loss of generality arises from assuming $x_{k}(p)=x_{k}(0), p \in[-d, 0]$ on each trial.

To include actuator faults, write the control input vector as

$$
u_{k}(p)=\left[\begin{array}{llllll}
u_{k 1}(p) & u_{k 2}(p) & \cdots & u_{k i}(p) & \cdots & u_{k m}(p)
\end{array}\right]^{T},
$$

and let

$$
u_{k}^{F}(p)=\left[\begin{array}{llllll}
u_{k 1}^{F}(p) & u_{k 2}^{F}(p) & \cdots & u_{k i}^{F}(p) & \cdots & u_{k m}^{F}(p)
\end{array}\right]^{T}
$$

represent the failed actuator with the following fault model, see, e.g., Tao, Paszke, Rogers, Yang, and Gałkowski (2017),

$$
u_{k i}^{F}(p)=\kappa_{i} u_{k i}(p), i=1,2, \ldots, m,
$$


where the actuator failure parameter $\kappa_{i}$ is unknown but satisfies the following condition

$$
0 \leq \underline{\kappa}_{i} \leq \kappa_{i} \leq \bar{\kappa}_{i}, i=1,2, \ldots, m \text {. }
$$

The parameters $\underline{\kappa}_{i}\left(\underline{\kappa}_{i} \leq 1\right), \bar{\kappa}_{i}\left(\bar{\kappa}_{i} \geq 1\right)$ in this fault model are assumed to be known. The fault-free case corresponds to $\kappa_{i}=1$ when $u_{k i}^{F}(p)=u_{k i}(p)$ and $\kappa_{i}=0$ corresponds to the outage case, i.e., complete failure to transmit a signal. The conditions $0<\underline{\kappa}_{i} \leq$ $\kappa_{i}<1$ and $1<\kappa_{i} \leq \bar{\kappa}_{i}$ correspond, respectively, to partial failures, e.g., partial degradation of an actuator or the abnormal case when the faulty actuator output is larger than the nominal controller output.

Introduce the matrices

$$
\begin{aligned}
& \bar{\kappa}=\operatorname{diag}\left\{\bar{\kappa}_{1}, \bar{\kappa}_{2}, \ldots, \bar{\kappa}_{m}\right\}, \underline{\kappa}=\operatorname{diag}\left\{\underline{\kappa}_{1}, \underline{\kappa}_{2}, \ldots, \underline{\kappa}_{m}\right\}, \\
& \kappa=\operatorname{diag}\left\{\kappa_{1}, \kappa_{2}, \ldots, \kappa_{m}\right\}
\end{aligned}
$$

and

$$
\begin{aligned}
\beta & =\operatorname{diag}\left\{\beta_{1}, \beta_{2}, \ldots, \beta_{m}\right\}, \beta_{0}=\operatorname{diag}\left\{\beta_{10}, \beta_{20}, \ldots, \beta_{m 0}\right\}, \\
\kappa_{0} & =\operatorname{diag}\left\{\kappa_{01}, \kappa_{02}, \ldots, \kappa_{0 m}\right\}, \\
\left|\kappa_{0}\right| & =\operatorname{diag}\left\{\left|\kappa_{01}\right|,\left|\kappa_{02}\right|, \ldots,\left|\kappa_{0 m}\right|\right\},
\end{aligned}
$$

where

$$
\beta_{i}=\frac{\left(\bar{\kappa}_{i}+\underline{\kappa}_{i}\right)}{2}, \beta_{i 0}=\frac{\bar{\kappa}_{i}-\underline{\kappa}_{i}}{\bar{\kappa}_{i}+\underline{\kappa}_{i}}, \kappa_{0 i}=\frac{\left(\kappa_{i}-\beta_{i}\right)}{\beta_{i}} .
$$

Then the matrix $\kappa$ can be rewritten into the following form

$$
\kappa=\left(I+\kappa_{0}\right) \beta
$$

and $\left|\kappa_{0}\right|$ satisfies

$$
\left|\kappa_{0}\right| \preceq \beta_{0} \preceq I .
$$

Since $\underline{\kappa}_{i}$ and $\bar{\kappa}_{i}$ are assumed to be known, an additional parameter $\beta$ in (4) is used to scale to the original range of the unknown scalars $\kappa_{i}$. Hence $\kappa$ in (3) is of the form (5) with a known $\beta_{0}$ and an unknown $\kappa_{0}$. Using these transformations, the model (1) results in

$$
\begin{aligned}
x_{k}(p+1)= & (A+\Delta A(p)) x_{k}(p)+\left(A_{d}+\Delta A_{d}(p)\right) x_{k}(p-d) \\
& +\kappa(B+\Delta B(p)) u_{k}(p), \\
y_{k}(p)= & C x_{k}(p) .
\end{aligned}
$$

\section{Robust ILC design}

Given the reference trajectory vector $y_{r}(p) \in \Re^{l}$, the tracking error on trial $k$, i.e., the difference between the desired and actual outputs, is

$$
e_{k}(p)=y_{r}(p)-y_{k}(p) \text {. }
$$


This tracking error is used to construct the input vector for next trial such that the sequence of tracking errors $\left\{e_{k}\right\}_{k>1}$ converges to zero, or to within some specified tolerance, as $k \rightarrow \infty$. (As detailed below convergence is defined in terms of a suitably chosen norm.)

A standard form of ILC law (i.e. the means of updating the control vector from trial-to trial), for a system described by (7) is

$$
u_{k+1}(p)=u_{k}(p)+r_{k+1}(p),
$$

where $r_{k+1}(p)$ is the correction term. This form of control law constructs the input for the next trial as the sum of the previous trial input and $r_{k+1}(p)$, where this last term is computed using the previous trial error. Introduce the vector

$$
\eta_{k+1}(p+1)=x_{k+1}(p)-x_{k}(p),
$$

where, without loss of generality, assume that $y_{r}(0)=y_{k}(0)=C x_{k}(0)$ and hence $\eta_{k}(0)=0$. This immediately implies that system returns to the same state initial vector at the start of each trial, where this choice is justified by many application areas, e.g., a gantry robot executing a pick and place task where resetting is to the same location. The question of uncertainty in the state initial vector is not considered and is left as an area for possible future research.

Assume that the correction term $r_{k+1}(p)$ of (8) takes the form

$$
r_{k+1}(p)=K_{1} \eta_{k+1}(p+1)+K_{2} e_{k}(p+1)
$$

where $K_{1}$ and $K_{2}$ are the control law matrices to be determined. This control law correction term is the sum of state feedback control based on $\eta_{k+1}$ plus a feedforward term based on the previous trial error $e_{k}$. Combining (8)-(10) gives the ILC dynamics state-space model

$$
\begin{aligned}
\eta_{k+1}(p+1) & =\hat{A}(p) \eta_{k+1}(p)+\hat{A}_{d}(p) \eta_{k+1}(p-d)+\hat{B}(p) e_{k}(p), \\
e_{k+1}(p) & =\hat{C}(p) \eta_{k+1}(p)+\hat{C}_{d}(p) \eta_{k+1}(p-d)+\hat{D}(p) e_{k}(p),
\end{aligned}
$$

where

$$
\begin{aligned}
\hat{A}(p) & =(A+\Delta A(p))+\kappa(B+\Delta B(p)) K_{1}, \hat{B}(p)=\kappa(B+\Delta B(p)) K_{2}, \\
\hat{C}(p) & =-C\left((A+\Delta A(p))+\kappa(B+\Delta B(p)) K_{1}\right), \\
\hat{D}(p) & =I-\kappa C(B+\Delta B(p)) K_{2}, \\
\hat{A}_{d}(p) & =A_{d}+\Delta A_{d}(p), \hat{C}_{d}(p)=-C\left(A_{d}+\Delta A_{d}(p)\right) .
\end{aligned}
$$

The state-space model (11) is a discrete linear repetitive process with current trial state vector $\eta_{k+1}$, zero input vector and previous pass profile vector $e_{k}$. In this model the terms $\hat{B}(p) e_{k}(p)$ and $\hat{D}(p) e_{k}(p)$ represent the contributions of the previous trial error to the current trial state and error respectively. This interaction is the source of the unique repetitive process control problem where the sequence $\left\{e_{k}\right\}_{k \geq 1}$ can contain oscillations that increase in amplitude from trial-to-trial, i.e., with increasing $k$.

The stability theory for linear repetitive processes Rogers et al. (2007) is formulated in terms of an abstract model of the dynamics in a Banach space setting where the 
trial-to-trial updating is represented by a bounded linear operator. Given the control problem, this theory requires that a bounded initial trial profile produces a bounded sequence of trial profiles $\left(\left\{e_{k}\right\}_{k \geq 1}\right.$ in the ILC setting) where boundedness is defined in terms of the norm on the underlying function space. Moreover, this property can be imposed over the finite and fixed trial length or for all possible values of this parameter, where the latter case can be analyzed mathematically by considering $\alpha \rightarrow \infty$. The former property is termed asymptotic stability and the latter stability along the trial.

Asymptotic stability forces $\left\{e_{k}\right\}$ to converge to a limit $e_{\infty}$ i.e., the ILC error converges from trial-to-trial but the finite trial length can result in a limit error that is unstable. Stability along the trial is required to prevent this problem from arising and for the ILC design considered, the following result is a direct application of the conditions for this property.

Lemma 4.1 (Rogers et al. (2007); Tao et al. (2017)). The discrete linear repetitive process (11) representing ILC dynamics is robustly stable along the trial $\forall d \in[0, \bar{d}]$ if, and only if

(1) $\rho(\hat{D}(p))<1$,

(2) $\rho\left(\hat{A}(p)+e^{-d j \theta} \hat{A}_{d}(p)\right)<1, \forall \theta \in[-\pi, \pi]$ and $d \in[0, \bar{d}]$,

(3) all eigenvalues of transfer-function matrix

$$
G\left(e^{j \theta}\right)=\left(\hat{C}(p)+e^{-d j \theta} \hat{C}_{d}(p)\right)\left(e^{j \theta} I-\hat{A}(p)-e^{-d j \theta} \hat{A}_{d}(p)\right)^{-1} \hat{B}(p)+\hat{D}(p),
$$

have modulus strictly less than unity $\forall \theta \in[-\pi, \pi]$ and $d \in[0, \bar{d}]$.

Note that the third condition of this last result requires frequency attenuation of the previous trial error dynamics over the complete frequency range. Alternatively, it can be expressed by requiring for each $\theta \in[-\pi, \pi]$ the existence of $P\left(e^{j \theta}\right) \succ 0$ and a scalar $\gamma$ satisfying $0<\gamma \leq 1$ such that

$$
G\left(e^{j \theta}\right)^{*} P\left(e^{j \theta}\right) G\left(e^{j \theta}\right)-\gamma^{2} P\left(e^{j \theta}\right) \prec 0, \forall \theta \in[-\pi, \pi] .
$$

In this last condition, the dependence of $P\left(e^{j \theta}\right)$ on $\theta$ is unknown and hence this inequality cannot be solved.

One possible simplifying approach is to seek a solution for the constant matrix $P$ $\forall \theta \in[-\pi, \pi]$. However, this approach can be conservative since a single matrix $P$ is used over the entire frequency range. The level of conservativeness can be reduced by dividing the entire frequency domain into sub-intervals and applying the GKYP Lemma to each sub-interval, where a different matrix to $P$ can be used in each of these. Also, attention can be limited to $\theta \in[0, \pi]$ (instead of considering the entire frequency range $[-\pi, \pi]$ ).

Using results in Pipeleers and Vandenberghe (2011), the constant matrix for each sub-interval can be chosen as a symmetric matrix with real entries without additional conservatism. Also, divide the frequency range into $H$ sub-intervals (not necessarily containing the same number of frequencies) such that

$$
[0, \pi]=\bigcup_{h=1}^{H}\left[\theta_{h-1}, \theta_{h}\right],
$$


where $\theta_{0}=0$ and $\theta_{H}=\pi$. Then condition (iii) of Theorem 4.1, i.e.,

$$
\rho\left(G\left(e^{j \theta}\right)\right)<1, \forall \theta \in[-\pi, \pi]
$$

can be replaced by

$$
\rho\left(G\left(e^{j \theta}\right)\right)<1, \forall \theta \in\left[\theta_{h-1}, \theta_{h}\right], h=1,2, \ldots, H .
$$

Since $H$ frequency sub-intervals are considered, the resulting process in (11) must be stable along the trial in each frequency sub-interval defined by (13).

The design problem considered is formulated as follows.

Given an ILC scheme described as a discrete linear repetitive process of the form (11) under an actuator fault of the form (3)-(6) and the entire frequency range arbitrarily divided into $H$ possible different frequency sub-intervals as in (13), determine the control law matrices $K_{1}$ and $K_{2}$ in (10) such that the resulting process is stable along the trial in each frequency sub-interval and hence forces the trial-to-trial error convergence to zero, i.e., $\lim _{k \rightarrow \infty}\left\|e_{k}\right\|=0, \lim _{k \rightarrow \infty}\left\|u_{k}-u_{\infty}\right\|$, where $\|\cdot\|$ denotes the norm on the underlying function space.

The analysis in the remainder of this paper makes use of a version of Theorem 1 in Zhang and Yang (2011) for the single delay case, including the following matrices Low frequency range: $|\theta|<\theta_{1}$

$$
\Psi=\left[\begin{array}{cc}
0 & 1 \\
1 & -2 \cos \left(\theta_{1}\right.
\end{array}\right]
$$

Middle frequency range: $\theta_{h-1} \leq \theta \leq \theta_{h}$

$$
\Psi=\left[\begin{array}{cc}
0 & e^{j\left(\frac{\theta_{h-1}+\theta_{h}}{2}\right)} \\
e^{-j\left(\frac{\theta_{h-1}+\theta_{h}}{2}\right)} & -2 \cos \left(\frac{\theta_{h}+\theta_{h-1}}{2}\right)
\end{array}\right]
$$

High frequency range, $|\theta|>\theta_{H-1}$

$$
\Psi=\left[\begin{array}{cc}
0 & -1 \\
-1 & 2 \cos \left(\theta_{H-1}\right)
\end{array}\right]
$$

Lemma 4.2. For a given real symmetric matrix $\Pi$ of compatible dimensions and any delay $d$ satisfying $0<d \leq \bar{d}$, a transfer-function matrix $G\left(e^{j \theta}\right)$ defined by (12) satisfies (14) over a specified frequency interval $\Theta$ if there exist $P \succ 0, Z \succ 0, Q \succ 0$ 
and $X \succ 0$, such that

$$
\begin{aligned}
& {\left[\begin{array}{ccc}
\hat{A}(p) & \hat{A}_{d}(p) & \hat{B}(p) \\
I & 0 & 0
\end{array}\right]^{T}\left(\Phi \otimes P+\Psi \otimes Q+\Psi_{0} \otimes \bar{d} Z\right)} \\
& \xi\left[\begin{array}{ccc}
\hat{A}(p) & \hat{A}_{d}(p) & \hat{B}(p) \\
I & 0 & 0
\end{array}\right] \\
& +\left[\begin{array}{ccc}
\hat{C}(p) & \hat{C}_{d}(p) & \hat{D}(p) \\
0 & 0 & I
\end{array}\right]^{T} \Pi\left[\begin{array}{ccc}
\hat{C}(p) & \hat{C}_{d}(p) & \hat{D}(p) \\
0 & 0 & I
\end{array}\right] \\
& +\left[\begin{array}{ccc}
X-\bar{d}^{-1} Z & \bar{d}^{-1} Z & 0 \\
\bar{d}^{-1} Z & -X-\bar{d}^{-1} Z & 0 \\
0 & 0 & 0
\end{array}\right] \prec 0 .
\end{aligned}
$$

Moreover, the following frequency domain inequality holds

$$
\left[\begin{array}{c}
G\left(e^{j \theta}\right) \\
I
\end{array}\right]^{*} \Pi\left[\begin{array}{c}
G\left(e^{j \theta}\right) \\
I
\end{array}\right] \prec 0, \forall \theta \in \Theta
$$

where

$$
\Phi=\left[\begin{array}{cc}
-1 & 0 \\
0 & 1
\end{array}\right], \Psi_{0}=\left[\begin{array}{cc}
1 & -1 \\
-1 & 1
\end{array}\right]
$$

and by (13), $\Theta$ is specified by the following choices for $\Psi$ in (16)-(18) ( recall that $\theta_{0}=0$ and $\left.\theta_{H}=\pi\right)$.

The analysis that follows in this paper uses the matrix

$$
\begin{aligned}
\Upsilon & =\left[\begin{array}{ll}
I & 0 \\
0 & I \\
0 & 0 \\
0 & 0
\end{array}\right] \Xi\left[\begin{array}{ll}
I & 0 \\
0 & I \\
0 & 0 \\
0 & 0
\end{array}\right]^{T}+\left[\begin{array}{cc}
0 & 0 \\
\hat{C}^{T}(p) & 0 \\
\hat{C}_{d}^{T}(p) & 0 \\
\hat{D}^{T}(p) & I
\end{array}\right] \Pi\left[\begin{array}{cc}
0 & 0 \\
\hat{C}^{T}(p) & 0 \\
\hat{C}_{d}^{T}(p) & 0 \\
\hat{D}^{T}(p) & I
\end{array}\right]^{T} \\
& +\left[\begin{array}{cccc}
0 & 0 & 0 & 0 \\
0 & X-\bar{d}^{-1} Z & \bar{d}^{-1} Z & 0 \\
0 & \bar{d}^{-1} Z & -X-\bar{d}^{-1} Z & 0 \\
0 & 0 & 0 & 0
\end{array}\right],
\end{aligned}
$$

where $\Xi=\left(\Phi \otimes P+\Psi \otimes Q+\Psi_{0} \otimes \bar{d} Z\right)$ is the only matrix whose block entries depend on the chosen frequency range - see (16-(18). Also, partition the matrix variable $\Xi$ as

$$
\Xi=\Phi \otimes P+\Psi \otimes Q+\Psi_{0} \otimes \bar{d} Z=\left[\begin{array}{c|c}
\Xi_{1} & \Xi_{2} \\
\hline \Xi_{2}^{*} & \Xi_{3}
\end{array}\right]
$$

where 


$$
\left.\Xi=\begin{array}{c|c}
E_{1} & \Xi_{2} \\
\hline \Xi_{2}^{*} & \Xi_{3}
\end{array}\right]=\left[\begin{array}{c|c}
-P+\bar{d} Z & Q-\bar{d} Z \\
\hline Q-d Z & P-2 \cos \left(\theta_{1}\right) Q+d Z
\end{array}\right],
$$

for the low frequency range,

$$
\Xi=\left[\begin{array}{c|c}
\Xi_{1} & \Xi_{2} \\
\hline \Xi_{2}^{*} & \Xi_{3}
\end{array}\right]=\left[\begin{array}{c|c}
-P+\bar{d} Z & e^{j \theta_{c}} Q-\bar{d} Z \\
\hline e^{-j \theta_{c}} Q-\bar{d} Z & P-2 \cos \left(\theta_{m}\right) Q+\bar{d} Z
\end{array}\right],
$$

for the middle frequency range,

$$
\Xi=\left[\begin{array}{c|c}
\Xi_{1} & \Xi_{2} \\
\hline \Xi_{2}^{*} & \Xi_{3}
\end{array}\right]=\left[\begin{array}{c|c}
-P+\bar{d} Z & -Q-\bar{d} Z \\
\hline-Q-d Z & P+2 \cos \left(\theta_{H-1}\right) Q+d Z
\end{array}\right],
$$

for the high frequency range

and

$$
\theta_{c}=\frac{\theta_{h}+\theta_{h-1}}{2}, \theta_{m}=\frac{\theta_{h}-\theta_{h-1}}{2} .
$$

\section{Monotonic convergence over a finite frequency domain}

Condition (iii) of Theorem 4.1 gives that the ILC tracking error converges as $k \rightarrow \infty$, i.e. the error reduces between successive trials if, and only if, $\rho\left(G\left(e^{j \theta}\right)\right)<1$. However, practical experience shows (see, e.g., Longman (2000)) that some ILC laws have poor transients during the convergence process even if the above condition is satisfied (e.g., the tracking error may grow over a number of trials). Considering this aspect, a stronger convergence criteria is required and one way of ensuring that the Euclidean norm of the tracking error decreases monotonically for each trial is to require that $G\left(e^{j \theta}\right)$ satisfies

$$
\bar{\sigma}\left(G\left(e^{j \theta}\right)\right)<1, \forall \theta \in[0, \pi]
$$

where $\bar{\sigma}(\cdot)$ denotes the maximum singular value of its matrix argument. Also

$$
\bar{\sigma}\left(G\left(e^{j \theta}\right)\right)<1 \Leftrightarrow\left\|G\left(e^{j \theta}\right)\right\|_{\infty}<1, \forall \theta \in[0, \pi] .
$$

Since this paper is concerned with the minimization of the $\mathcal{H}_{\infty}$ norm in frequency intervals, matrix $\Pi$ in (20) is selected as

$$
\Pi=\left[\begin{array}{cc}
I & 0 \\
0 & -\gamma^{2} I
\end{array}\right],
$$

where $\gamma$ is a given scalar that satisfies $0<\gamma \leq 1$. Additionally, let $\|\cdot\|_{2}$ denote the $\ell_{2}$ norm and then

$$
\left\|e_{k}(p)\right\|_{2} \leq\|G(z)\|_{\infty}^{k}\left\|e_{0}(p)\right\|_{2} .
$$


Therefore if

$$
\left\|G\left(e^{j \theta}\right)\right\|_{\infty}<\gamma, \forall \theta \in[0, \pi]
$$

monotonic trial-to-trial error convergence, i.e., $\left\|e_{k+1}\right\|<\left\|e_{k}\right\|, k \geq 1$, occurs in $\ell_{2}$ for $k \rightarrow \infty$.

Consider the case when the performance requirements are specified over a finite set, say $\Theta$. In particular, it is required to impose different specifications over a low frequency range specified by $0 \leq|\theta| \leq \theta_{1}$, a middle frequency range specified by $\theta_{h-1} \leq \theta \leq \theta_{h}$ and a high frequency range specified by $|\theta| \geq \theta_{H-1} \leq \pi$. Then in such a case, (28) is modified to

$$
\left\|G\left(e^{j \theta}\right)\right\|_{\infty}<\gamma, \forall \theta \in \Theta
$$

where $\Theta$ is a finite frequency range of interest in a given application. (See Paszke et al. (2016) for results with experimental verification for discrete systems without time delays and fault free where the design is based on this last condition.)

The following new result gives a test to verify if stability along the trial over finite frequency interval holds and is central to the new results developed in this paper.

Theorem 5.1. An ILC scheme described as a discrete linear repetitive process of the form (11) under an actuator fault of the form (3)-(6) has i) the stability along the trial property over the finite frequency ranges defined in (16)-(18) and ii) monotonic trialto-trial error convergence occurs over the same frequency intervals and any delay $d$ satisfying $0<d \leq \bar{d}$ if for a given constant $\gamma \in(0,1]$ there exist matrices $P \succ 0$, $Q \succ 0, X \succ 0, Z \succ 0$ and $W$ such that $\Omega_{1} \prec 0$ where

$$
\Omega_{1}=\left[\begin{array}{ccccc}
\Xi_{1} & \Xi_{2}-W & 0 & 0 & 0 \\
(\star) & \Xi_{3}+X-\bar{d}^{-1} Z+\operatorname{sym}\left\{W^{T} \hat{A}(p)\right\} & \bar{d}^{-1} Z+W^{T} \hat{A}_{d}(p) & W^{T} \hat{B}(p) & \hat{C}^{T}(p) \\
(\star) & (\star) & -X-\bar{d}^{-1} Z & 0 & \hat{C}_{d}^{T}(p) \\
(\star) & (\star) & (\star) & -\gamma^{2} I & \hat{D}^{T}(p) \\
(\star) & (\star) & (\star) & (\star) & -I
\end{array}\right],
$$

where the matrices $\Xi_{1}, \Xi_{2}$ and $\Xi_{3}$ are, respectively, given by (23), (24) and (25).

Proof. Suppose that (29) is feasible for some $P \succ 0, Q \succ 0, X \succ 0, Z \succ 0$ and $W$. Therefore $\hat{D}^{T} \hat{D}-\gamma^{2} I \prec 0$ and condition $(i)$ of Theorem 4.1 must hold for $\gamma \in(0,1]$. After applying Schur's complement formula and defining

$$
\Lambda=\left[\begin{array}{llll}
-I & \hat{A}(p) & \hat{A}_{d}(p) & \hat{B}(p)
\end{array}\right], \Sigma=\left[\begin{array}{llll}
0 & I & 0 & 0
\end{array}\right]
$$

inequality (29) can be rewritten as

$$
\Upsilon+\operatorname{sym}\left\{\Lambda^{T} W \Sigma\right\} \prec 0,
$$


where $\Upsilon$ is defined in (21). Also, by construction, the matrices $\Lambda_{\perp}$ and $\Sigma_{\perp}$ are

$$
\Sigma_{\perp}=\left[\begin{array}{ccc}
I & 0 & 0 \\
0 & 0 & 0 \\
0 & I & 0 \\
0 & 0 & I
\end{array}\right], \Lambda_{\perp}=\left[\begin{array}{ccc}
\hat{A}(p) & \hat{A}_{d}(p) & \hat{B}(p) \\
I & 0 & 0 \\
0 & I & 0 \\
0 & 0 & I
\end{array}\right]
$$

and for this $\Sigma_{\perp}$, the inequality

$$
\begin{aligned}
& \Sigma_{\perp}^{T} \Upsilon \Sigma_{\perp} \\
& =\left[\begin{array}{ccc}
\Xi_{1} & 0 & 0 \\
(\star) & \hat{C}_{d}^{T}(p) \hat{C}_{d}(p)-X-\bar{d}^{-1} Z & \hat{C}_{d}^{T}(p) \hat{D}(p) \\
(\star) & (\star) & \hat{D}^{T}(p) \hat{D}(p)-\gamma^{2} I
\end{array}\right] \prec 0
\end{aligned}
$$

is feasible if (29) holds. Furthermore, the inequality (19) can be rewritten as

$$
\Lambda_{\perp}^{T} \Upsilon \Lambda_{\perp} \prec 0
$$

and by Lemma 2.2, feasibility of (29) implies feasibility of (19) for $\Pi$ given by (27). Hence the condition ( iii) of Theorem 4.1 holds. Moreover, the inequality (19) requires $P \succ 0$ and $X \succ 0$ to guarantee that $\rho\left(\hat{A}+e^{-j \theta d} \hat{A}_{d}\right)<1$ Zhang and Yang (2011) for any delay $d$ satisfying $0<d \leq \bar{d}$ and therefore condition (ii) of Theorem 4.1 also holds. Hence, stability along the trial over the specified frequency range holds and the proof is complete.

\section{LMI-based control law design}

Given the result of Theorem 5.1, the design problem can be formulated as the search for some matrix variables. Unfortunately, this formulation is not directly useful for ILC control law design because the inequalities (29) are not LMIs as they are bilinear in $W$ and the matrices $K_{1}$ and $K_{2}$ defining the ILC law correction term (10). Hence no computationally effective method exists for checking (29). This section develops a method to re-parameterize the inequalities (29) such that the problem is equivalently converted to an LMI design condition.

To proceed, consider the nominal model case $\left(\Delta A(p)=0, \Delta A_{d}(p)=0, \Delta B(p)=0\right)$ for which the following result gives an LMI condition for stability along the trial over the frequency range $\Theta$.

Theorem 6.1. An ILC scheme described as a discrete linear repetitive process of the form (11) in the absence of uncertainty and under an actuator fault of the form (3)(6) has i) the stability along the trial property over the finite frequency ranges defined in (16)-(18) and ii) monotonic trial-to-trial error convergence occurs over the same frequency intervals and any delay $d$ satisfying $0<d \leq \bar{d}$, if for a given constant $\gamma \in(0,1]$, there exist matrices $\hat{P} \succ 0, \hat{Q} \succ 0, \hat{X} \succ 0, \hat{Z} \succ 0, S, L$ and a scalar $\varepsilon_{1}>0$, 
such that $\Omega_{1} \prec 0$, where

$$
\Omega_{1}=\left[\begin{array}{ccccccc}
\hat{\Xi}_{1} & \hat{\Xi}_{2}-S^{T} & 0 & 0 & 0 & 0 & 0 \\
(\star) & T_{1} & A_{d} S+\bar{d}^{-1} \hat{Z} & \beta B L & -\left(C A S+\beta C B X_{1}\right)^{T} & \varepsilon_{1} \beta_{0} B & \beta X_{1}^{T} \\
(\star) & (\star) & -\hat{X}-\bar{d}^{-1} \hat{Z} & 0 & -\left(C A_{d} S\right)^{T} & 0 & 0 \\
(\star) & (\star) & (\star) & -\gamma^{2} I & (I-\beta C B L)^{T} & 0 & \beta L^{T} \\
(\star) & (\star) & (\star) & (\star) & -I & -\varepsilon_{1} \beta_{0} C B & 0 \\
(\star) & (\star) & (\star) & (\star) & (\star) & -\varepsilon_{1} I & 0 \\
(\star) & (\star) & (\star) & (\star) & (\star) & (\star) & -\varepsilon_{1} I
\end{array}\right],
$$

holds, where $T_{1}=\hat{\Xi}_{3}+\hat{X}-\bar{d}^{-1} \hat{Z}+\operatorname{sym}\left\{A S+\beta B X_{1}\right\}$ and $\hat{\Xi}_{1}, \hat{\Xi}_{2}$ and $\hat{\Xi}_{3}$ have the same form as the matrices $\Xi_{1}, \Xi_{2}$ and $\Xi_{3}$ (see (23)-(25)) respectively but with $P, Q$, and $Z$ replaced, respectively, by $\hat{P}, \hat{Q}$, and $\hat{Z}$. Moreover, if this LMI is feasible, the corresponding matrices in the ILC law (10) are given by

$$
K_{1}=X_{1} S^{-1}, K_{2}=L
$$

Proof. Suppose that there exist $\hat{P} \succ 0, \hat{Q} \succ 0, \hat{X} \succ 0, \hat{Z} \succ 0, S, L$ and a scalar $\varepsilon_{1}>0$, such that the LMI of (33) is feasible. Then application of the Schur's complement formula to (33) gives

$$
\Omega_{2}+\varepsilon_{1} \beta_{0}^{2} H_{1} H_{1}^{T}+\varepsilon_{1}^{-1} F_{1}^{T} F_{1} \prec 0
$$

where

$$
\begin{aligned}
\Omega_{2} & =\left[\begin{array}{ccccc}
\hat{\Xi}_{1} & \hat{\Xi}_{2}-S^{T} & 0 & 0 & 0 \\
(\star) & \mathrm{T}_{1} & A_{d} S+\bar{d}^{-1} \hat{Z} & \beta B L & -\left(C A S+\beta C B X_{1}\right)^{T} \\
(\star) & (\star) & -\hat{X}-\bar{d}^{-1} \hat{Z} & 0 & -\left(C A_{d} S\right)^{T} \\
(\star) & (\star) & (\star) & -\gamma^{2} I & (I-\beta C B L)^{T} \\
(\star) & (\star) & (\star) & (\star) & -I
\end{array}\right], \\
H_{1} & =\left[\begin{array}{c}
0 \\
B \\
0 \\
0 \\
-C B
\end{array}\right], F_{1}^{T}=\left[\begin{array}{c}
0 \\
\beta X_{1}^{T} \\
0 \\
\beta L^{T} \\
0
\end{array}\right] .
\end{aligned}
$$

Using (6) and applying Lemma 2.1, this last inequality is transformed to

$$
\Omega_{2}+\operatorname{sym}\left\{H_{1} \kappa_{0} F_{1}\right\} \prec 0 \text {. }
$$

Next, apply the congruence transformation specified by diag, $\left\{S^{-1}, S^{-1}, S^{-1}, I, I\right\}$ to this last inequality and set $W=S^{-1}, \Xi_{1}=S^{-T} \hat{\Xi}_{1} S^{-1}, \Xi_{2}=S^{-T} \hat{\Xi}_{2} S^{-1}, \Xi_{3}=$ $S^{-T} \hat{\Xi}_{3} S^{-1}, X=S^{-T} \hat{X} S^{-1}, Z=S^{-T} \hat{Z} S^{-1}, Q=S^{-T} \hat{Q} S^{-1}, P=S^{-T} \hat{P} S^{-1}$ to 
obtain

$$
\left[\begin{array}{ccccc}
\Xi_{1} & \Xi_{2}-W & 0 & 0 & 0 \\
(\star) & T_{2} & \bar{d}^{-1} Z+W^{T} A_{d} & W^{T} \kappa B K_{2} & \left(-C\left(A+\kappa B K_{1}\right)\right)^{T} \\
(\star) & (\star) & -X-\bar{d}^{-1} Z & 0 & -C A_{d}^{T} \\
(\star) & (\star) & (\star) & -\gamma^{2} I & \left(I-\kappa C B K_{2}\right)^{T} \\
(\star) & (\star) & (\star) & (\star) & -I
\end{array}\right] \prec 0
$$

where $T_{2}=\Xi_{3}+X-\bar{d}^{-1} Z+\operatorname{sym}\left\{W^{T}\left(A+\kappa B K_{1}\right)\right\}$. Finally, the inequality (35) is a version of (29) and hence analogous reasoning can be followed to complete the proof.

Remark 1. To increase the speed of the monotonic trial-to-trial convergence rate, the scalar $\gamma$ can be minimized by solving the following linear minimization problem

$$
\begin{aligned}
& \min _{\hat{P} \succ 0, \hat{Q} \succ 0, \hat{X} \succ 0, \hat{Z} \succ 0, S, L, \varepsilon_{1}>0, \mu>0} \mu \\
& \text { subject to (33) with } \mu=\gamma^{2} .
\end{aligned}
$$

Consider the case when uncertainty is present, i.e., the matrices $\Delta A(p), \Delta A_{d}(p)$ and $\Delta B(p)$ are present in (1) and are of the form (2). Hence, the inequality in Theorem 6.1 will include terms formed by multiplication of two matrices with additive uncertainties. The next result reformulates the condition of Theorem 6.1 to an LMIbased characterization of the robust monotonic trial-to-trial error convergence.

Theorem 6.2. An ILC scheme described as a discrete linear repetitive process of the form (11) in the absence of uncertainty and under an actuator fault of the form (3)(6) has i) the stability along the trial property over the finite frequency ranges defined in (16)-(18) and ii) monotonic trial-to-trial error convergence occurs over the same frequency intervals and any delay $d$ satisfying $0<d \leq \bar{d}$ if, for a given constant $\gamma \in(0,1]$, there exist matrices $\hat{P} \succ 0, \hat{Q} \succ 0, \hat{X} \succ 0, \hat{Z} \succ 0, S, L$ and positive scalars $\varepsilon_{1}$ and $\lambda_{1}$ such that

$$
\left[\begin{array}{ccc}
\Omega_{1} & \lambda_{1} H_{2}^{T} & F_{2}^{T} \\
(\star) & -\lambda_{1} I & 0 \\
(\star) & (\star) & -\lambda_{1} I
\end{array}\right] \prec 0,
$$

where $\Omega_{1}$ is given by (33) and

$$
\begin{aligned}
H_{2} & =\left[\begin{array}{ccccccc}
0 & E_{1}^{T} & 0 & 0 & \left(-C E_{1}\right)^{T} & 0 & 0 \\
0 & E_{2}^{T} & 0 & 0 & \left(-C E_{2}\right)^{T} & 0 & 0
\end{array}\right], \\
F_{2} & =\left[\begin{array}{cccccccc}
0 & F_{1} S & F_{d} S & 0 & 0 & 0 & 0 \\
0 & \beta F_{2} X_{1} & 0 & \beta F_{2} L & 0 & \varepsilon_{1} \beta_{0} F_{2} & 0
\end{array}\right] .
\end{aligned}
$$

Moreover, if the LMI of (36) is feasible, the control law matrices $K_{1}$ and $K_{2}$ are given by (34).

Proof. Suppose that the LMI (36) is feasible. Then application of Schur's complement formula to (36) gives

$$
\Omega_{1}+\lambda_{1} H_{2}^{T} H_{2}+\lambda_{1}^{-1} F_{2}^{T} F_{2} \prec 0
$$


and by Lemma 2.1 this last inequality is feasible if and only if

$$
\Omega_{1}+\operatorname{sym}\left\{H_{2}^{T} \delta(p) F_{2}\right\} \prec 0 .
$$

The last inequality is (33) applied to the uncertain case and by the result of Theorem 6.1 , feasibility of (36) ensures that a discrete linear repetitive process of the form (11) is robustly stable along the trial. This implies the robust monotonic trialto-trial error convergence and the proof is complete.

\subsection{Output information based ILC law design}

The control law (10) is state feedback based and therefore assumes that all entries in the state vector on any trial are available for measurement. If this is not the case, one option is to use a state observer. Another alternative is to use control law that only uses measured output information, i.e., the trial profile, and design of such a control law is the subject of this section. Specifically, the correction to the control law has the form

$$
r_{k+1}(p)=\bar{K}_{1} \mu_{k+1}(p+1)+K_{2} e_{k}(p+1),
$$

where

$$
\mu_{k+1}(p+1)=y_{k+1}(p)-y_{k}(p)=C \eta_{k+1}(p+1),
$$

and $\bar{K}_{1}$ and $K_{2}$ are compatibly dimensioned control law matrices to be designed. Application of (37) results in ILC dynamics described by

$$
\begin{aligned}
\eta_{k+1}(p+1) & =\bar{A}(p) \eta_{k+1}(p)+\hat{A}_{d}(p) \eta_{k+1}(p-d)+\hat{B}(p) e_{k}(p), \\
e_{k+1}(p) & =\bar{C}(p) \eta_{k+1}(p)+\hat{C}_{d}(p) \eta_{k+1}(p-d)+\hat{D}(p) e_{k}(p),
\end{aligned}
$$

where the matrices $\hat{A}_{d}(p), \hat{B}(p), \hat{C}_{d}(p), \hat{D}(p)$ are again given in (11) and

$$
\begin{aligned}
\bar{A}(p) & =(A+\Delta A(p))+\kappa(B+\Delta B(p)) \bar{K}_{1} C, \bar{C}(p) \\
& =-C\left((A+\Delta A(p))+\kappa(B+\Delta B(p)) \bar{K}_{1} C\right) .
\end{aligned}
$$

Defining a new matrix variable $R$ as $R=\bar{K}_{1} C-K_{1}$, where $K_{1}$ is a state feedback gain matrix that stabilizes (i.e., ensures stability along the trial) the dynamics, gives

$$
(B+\Delta B(p)) \bar{K}_{1} C=(B+\Delta B(p)) K_{1}+(B+\Delta B(p)) R .
$$

Then, based on the results developed in the previous section, i.e. by considering that there exist stabilizing matrices $K_{1}$ and $K_{2}$, an effective method for computing the matrix $\bar{K}_{1}$ such that the controlled process is stable along the trial over the frequency range $\Theta$ can be obtained. As in the previous section of this paper, the nominal case, (i.e., $\Delta A(p)=0, \Delta A_{d}(p)=0, \Delta B(p)=0$ is considered first).

Theorem 6.3. Suppose that the control law matrices $K_{1}$ and $K_{2}$ given in (34) have already been computed. Then an ILC scheme described as a discrete linear repetitive process of the form (11) in the absence of uncertainty and under an actuator fault of 
the form (3)-(6) has i) the stability along the trial property over the finite frequency ranges defined in (16)-(18) and ii) monotonic trial-to-trial error convergence occurs over the same frequency intervals and any delayd satisfying $0<d \leq \bar{d}$ if, for a given constant $\gamma \in(0,1]$, there exist matrices $P \succ 0, Q \succ 0, X \succ 0, Z \succ 0, W, M, N$ and a scalar $\varepsilon_{1}>0$, such that

$$
\Omega_{5}=\left[\begin{array}{cc}
\bar{H}_{1} & \bar{H}_{2}
\end{array}\right] \prec 0,(39)
$$

where

$$
\begin{gathered}
{\left[\begin{array}{ccccc}
\Xi_{1} & \Xi_{2}-W & 0 & 0 & 0 \\
(\star) & T_{3} & W^{T} A_{d}+\bar{d}^{-1} Z & \beta W^{T} B K_{2} & -\left(C A+\beta C B K_{1}\right)^{T} \\
(\star) & (\star) & -X-\bar{d}^{-1} Z & 0 & -\left(C A_{d}\right)^{T} \\
(\star) & (\star) & (\star) & -\gamma^{2} I & \left(I-\beta C B K_{2}\right)^{T} \\
(\star) & (\star) & (\star) & (\star) & -I \\
(\star) & (\star) & (\star) & (\star) & (\star) \\
(\star) & (\star) & (\star) & (\star) & (\star)
\end{array}\right],} \\
\bar{H}_{2}=\left[\begin{array}{ccc}
0 & 0 & \\
T_{4}+\beta W^{T} B & \varepsilon_{1} \beta_{0} W^{T} B & \beta K_{1}^{T} \\
0 & 0 & 0 \\
0 & 0 & \beta K_{2}^{T} \\
T_{4}-\beta C B & -\varepsilon_{1} \beta_{0} C B & 0 \\
(\star) & (\star) & (\star) \\
(\star) & (\star) & -N-N^{T} \\
0 & \beta I & \\
0 & -\varepsilon_{1} I & 0 \\
(\star) & 0 & -\varepsilon_{1} I
\end{array}\right]
\end{gathered}
$$

where $T_{3}=\Xi_{3}+X-\bar{d}^{-1} Z+\operatorname{sym}\left\{W^{T}\left(A+\beta B K_{1}\right)\right\}, T_{4}=\left(M C-N K_{1}\right)^{T}$ and $\Xi_{1}$, $\Xi_{2}$ and $\Xi_{3}$ are defined by (23)-(25). Moreover, if this last LMI is feasible for given $K_{1}$ and $K_{2}$ then the matrix $\bar{K}_{1}$ in the ILC law (37) is given by

$$
\bar{K}_{1}=N^{-1} M .
$$

Proof. Suppose that there exist $P \succ 0, Q \succ 0, X \succ 0, Z \succ 0, W, M, N$ and a scalar $\varepsilon_{1}>0$, such that the LMI (39) is feasible for given $K_{1}$ and $K_{2}$ (where $K_{1}$ and $K_{2}$ are computed a priori using Theorem 6.1). Moreover, $N+N^{T} \succ 0$, which implies that $N$ is invertible. Next, routine application of Schur's complement formula enables the LMI (39) to be written as

$$
\Omega_{3}+\varepsilon_{1} \beta_{0}^{2} H_{3} H_{3}^{T}+\varepsilon_{1}^{-1} F_{3}^{T} F_{3} \prec 0
$$

where $F_{3}=\left[\begin{array}{llllll}0 & \beta K_{1} & 0 & \beta K_{2} & 0 & \beta I\end{array}\right]$ and $\Omega_{3}$ and $H$ are given next. 


$$
\Omega_{3}=\left[\begin{array}{cccccc}
\Xi_{1} & \Xi_{2}-W & 0 & 0 & 0 & 0 \\
(\star) & T_{3} & W^{T} A_{d}+\bar{d}^{-1} Z & \beta W^{T} B K_{2} & -\left(C A+\beta C B K_{1}\right)^{T} & T_{4}+\beta W^{T} B \\
(\star) & (\star) & -X-\bar{d}^{-1} Z & 0 & -\left(C A_{d}\right)^{T} & 0 \\
(\star) & (\star) & (\star) & -\gamma^{2} I & \left(I-\beta C B K_{2}\right)^{T} & 0 \\
(\star) & (\star) & (\star) & (\star) & -I & T_{4}-\beta C B \\
(\star) & (\star) & (\star) & (\star) & (\star) & -N-N^{T}
\end{array}\right]
$$

$$
\mathrm{H}_{3}=\left[\begin{array}{c}
0 \\
W^{T} B \\
0 \\
0 \\
-C B \\
0
\end{array}\right]
$$

Using Lemma 2.1 and (6), this last inequality can be written as

$$
\left[\begin{array}{cccccc}
\Xi_{1} & \Xi_{2}-W & 0 & 0 & 0 & 0 \\
(\star) & T_{2} & \bar{d}^{-1} Z+W^{T} A_{d} & W^{T} \kappa B K_{2} & \left(-C\left(A+\kappa B K_{1}\right)\right)^{T} & T_{4}+\kappa W^{T} B \\
(\star) & (\star) & -X-\bar{d}^{-1} Z & 0 & -C A_{d}^{T} & 0 \\
(\star) & (\star) & (\star) & -\gamma^{2} I & \left(I-\kappa C B K_{2}\right)^{T} & 0 \\
(\star) & (\star) & (\star) & (\star) & -I & T_{4}-\kappa C B \\
(\star) & (\star) & (\star) & (\star) & (\star) & -N-N^{T}
\end{array}\right] \prec 0,
$$

where $T_{2}$ is defined as in (35). Also, introduce the matrices

$$
\begin{aligned}
\Omega_{4} & =\left[\begin{array}{ccccc}
\Xi_{1} & \Xi_{2}-W & 0 & 0 & 0 \\
(\star) & T_{2} & \bar{d}^{-1} Z+W^{T} A_{d} & W^{T} \kappa B K_{2} & \left(-C\left(A+\kappa B K_{1}\right)\right)^{T} \\
(\star) & (\star) & -X-\bar{d}^{-1} Z & 0 & -C A_{d}^{T} \\
(\star) & (\star) & (\star) & -\gamma^{2} I & \left(I-\kappa C B K_{2}\right)^{T} \\
(\star) & (\star) & (\star) & (\star) & -I
\end{array}\right] \\
H_{4} & =\left[\begin{array}{c}
0 \\
R^{T} \\
0 \\
0 \\
R^{T}
\end{array}\right], F_{4}^{T}=\left[\begin{array}{c}
0 \\
\kappa B^{T} W \\
0 \\
-\kappa C B \\
0
\end{array}\right]
\end{aligned}
$$

to rewrite (41) as

$$
\left[\begin{array}{cc}
\Omega_{4} & F_{4}^{T}+H_{4} N^{T} \\
F_{4}+N H_{4}^{T} & -N-N^{T}
\end{array}\right] \prec 0 .
$$

Moreover, it follows that (42) can be rewritten as

$$
\left[\begin{array}{cc}
\Omega_{4} & F_{4}^{T} \\
F_{4} & 0
\end{array}\right]+\operatorname{sym}\left\{\left[\begin{array}{l}
0 \\
I
\end{array}\right] N\left[\begin{array}{ll}
H_{4}^{T} & -I
\end{array}\right]\right\} \prec 0 .
$$


Also $\Omega_{4} \prec 0$ holds if $K_{1}$ and $K_{2}$ are known stabilizing control law matrices and a direct application of Lemma 2.2 gives

$$
\Omega_{4}+\operatorname{sym}\left\{F_{4}^{T} H_{4}^{T}\right\} \prec 0
$$

and it follows immediately that the previous inequality is equivalent to

$$
\left[\begin{array}{ccccc}
\Xi_{1} & \Xi_{2}-W & 0 & 0 & 0 \\
(\star) & T_{5} & \bar{d}^{-1} Z+W^{T} A_{d} & W^{T} \kappa B K_{2} & \left(-C\left(A+\kappa B \bar{K}_{1} C\right)\right)^{T} \\
(\star) & (\star) & -X-\bar{d}^{-1} Z & 0 & -C A_{d}^{T} \\
(\star) & (\star) & (\star) & -\gamma^{2} I & \left(I-\kappa C B K_{2}\right)^{T} \\
(\star) & (\star) & (\star) & (\star) & -I
\end{array}\right] \prec 0,
$$

where $T_{5}=\Xi_{3}+X-\bar{d}^{-1} Z+\operatorname{sym}\left\{W^{T}\left(A+\kappa B \bar{K}_{1} C\right)\right\}$. Finally, the last inequality is only satisfied when $\bar{K}_{1}$ is given by (40) and the proof is complete.

The solution to the state feedback design problem in the first stage of this last control law design procedure is non-unique. The desired performance (depending on the chosen strategy, e.g., minimizing $\gamma$ ) can be implemented using the following general iterative procedure.

(1) Calculate the required $K_{1}$ and $K_{2}$ using Theorem 6.1, i.e., solve the LMI (33).

(2) Using the matrices computed in the previous step, compute the control law matrix $\bar{K}_{1}$ by applying the result of Theorem 6.3 .

(3) Check if the required control performance index level (or maximal tolerable number of iterations) is obtained. If not, calculate $K_{1}=\bar{K}_{1} C$ and return to step 2 of this procedure. In the case of success, exit the loop and $\bar{K}_{1}$ is the desired control law matrix.

\subsection{Design in the presence of uncertainty}

Consider the case when uncertainty is present, i.e., the matrices $\Delta A(p), \Delta A_{d}(p)$ and $\Delta B(p)$ are present in (38) and are of the form (2). Then the following result gives an LMI-based characterization of the robust monotonic trial-to-trial error convergence under an output control law.

Theorem 6.4. Suppose that the control law matrices $K_{1}$ and $K_{2}$ have already been computed. Then an ILC scheme described as a discrete linear repetitive process of the form (11) in the absence of uncertainty and under an actuator fault of the form (3)(6) has i) the stability along the trial property over the finite frequency ranges defined in (16)-(18) and ii) monotonic trial-to-trial error convergence occurs over the same frequency intervals and any delay $d$ satisfying $0<d \leq \bar{d}$ if, for a given constant $\gamma \in(0,1]$, there exist matrices $P \succ 0, Q \succ 0, X \succ 0, Z \succ 0, W, M, N$ and positive scalars $\varepsilon_{1}$ and $\lambda_{1}$ such that

$$
\left[\begin{array}{ccc}
\Omega_{5} & \lambda_{1} H_{5}^{T} & F_{5}^{T} \\
(\star) & -\lambda_{1} I & 0 \\
(\star) & (\star) & -\lambda_{1} I
\end{array}\right] \prec 0,
$$


where $\Omega_{5}$ is defined in (39) and

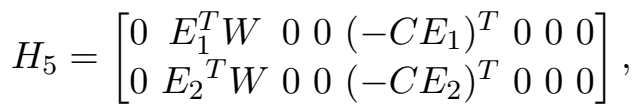

$$
\begin{aligned}
& F_{5}=\left[\begin{array}{cccccccc}
0 & F_{1} & F_{d} & 0 & 0 & 0 & 0 & 0 \\
0 & \beta F_{2} K_{1} & 0 & \beta F_{2} K_{2} & 0 & \beta F_{2} & \varepsilon_{1} \beta_{0} F_{2} & 0
\end{array}\right] .
\end{aligned}
$$

If this LMI is feasible for given $K_{1}$ and $K_{2}$, then the corresponding control law matrix $\bar{K}_{1}$ in the ILC law (37) is given by (40).

Proof. Suppose that LMI (43) is feasible for some $P \succ 0, Q \succ 0, X \succ 0, Z \succ 0, W$, $M, N$ and positive scalars $\varepsilon_{1}$ and $\lambda_{1}$. Then application of Schur's complement formula to (43) gives

$$
\Omega_{5}+\lambda_{1} H_{5}^{T} H_{5}+\lambda_{1}^{-1} F_{5}^{T} F_{5} \prec 0
$$

and by Lemma 2.1 this inequality is feasible if and only if

$$
\Omega_{5}+\operatorname{sym}\left\{H_{5}^{T} \delta(p) F_{5}\right\} \prec 0 .
$$

This last inequality is (39) applied to the uncertainty case and by the result of Theorem 6.1 feasibility of (43) ensures that a discrete linear repetitive process of the form (38) is robustly stable along the trial and the proof is complete.

\subsection{Practical implementation issues}

For applications, a critical problem is to achieve the desired shape of $\bar{\sigma}\left(G\left(e^{j \theta}\right)\right)$ over the complete frequency range, i.e., $[0, \pi]$. In particular, the possibility to impose different performance specifications has considerable practical significance since common performance issues occur over different frequency ranges. For example, the trial-totrial error convergence rate is in the 'low' frequency range whereas low sensitivity to disturbances and sensor noise is at frequencies above this range.

The LMI condition in the new design result in this paper can be applied over these frequency intervals once they have been chosen. In particular, the control performance for low frequency range is imposed over the first interval, i.e., $h=1$ and those for the high frequency range over the last interval, i.e., $h=H$. The specifications for the middle frequency range can be defined over remaining intervals for $2 \leq h \leq H-1$.

A practically feasible approach for the partitioning of the entire frequency range is to cover the reference signal bandwidth with the first frequency interval $(h=1)$. This frequency range is of primary interest because these frequencies have the most effect on the speed of the trial-to-trial error convergence. Additionally, by minimizing the attenuation level $\gamma$ in this frequency range a higher speed of monotonic trial-to-trial error convergence is obtained. The second frequency range frequently should cover these frequencies where the plant model accurately fits real response of the plant and hence the control is effective. In the last frequency range (for $h=H$ ), $\gamma \ll 1$ is required since this mitigates modeling errors and non-repetitive disturbances.

The last issue is to solve the problem when the control specifications cannot be satisfied over some frequency ranges. This means that the learning has to be performed over those frequencies where the LMI condition of Theorem 6.1 is satisfied and the re- 
maining frequencies cut-off by a low-pass filter with cut-off frequency equal to the highest frequency, for which the result of Theorem 6.1 is valid. A zero-phase filter can be used (all of the data is available when a trial is complete and the filter run between trials). For implementation, substituting in (10) for $\eta_{k+1}(p+1)$ and $e_{k}(p+1)$ gives the ILC law as

$$
u_{k+1}(p)=u_{k}(p)+K_{1}\left(x_{k+1}(p)-x_{k}(p)\right)+K_{2}\left(y_{r}(p+1)-y_{k}(p+1)\right) .
$$

\section{Simulation based Case Study}

To illustrate the effectiveness and feasibility of the new ILC design, this section gives the results of a numerical case study where (the delay-free part of this model was identified from measured data, see L. Wang, Mo, Qu, et al. (2013)) and the time-delay range is $0<d \leq \bar{d}=1$, where $d=1$ is used in the simulation results given below. The nominal model for ILC design is

$$
\begin{aligned}
x_{k}(p+1)= & \left(\left[\begin{array}{cc}
1.582 & -0.5916 \\
1 & 0
\end{array}\right]+\Delta A(p)\right) x_{k}(p) \\
& +\left(\left[\begin{array}{cc}
0.102 & 0.101 \\
0.203 & 0
\end{array}\right]+\Delta A_{d}(p)\right) x_{k}(p-d) \\
& +\left(\left[\begin{array}{l}
1 \\
0
\end{array}\right]+\Delta B(p)\right) u_{k}(p), \\
y_{k}(p)= & {[1.69 \quad 1.419] x_{k}(p) }
\end{aligned}
$$

and the time-varying uncertainties $\Delta A(p), \Delta A_{d}(p)$ and $\Delta B(p)$ of (2) are taken as

$$
\begin{aligned}
& E_{1}=\left[\begin{array}{ll}
1 & 0 \\
0 & 1
\end{array}\right], F_{1}=\left[\begin{array}{ll}
0.08 & 0 \\
0.08 & 0
\end{array}\right], F_{2}=\left[\begin{array}{c}
0.1 \\
0.14
\end{array}\right], \\
& E_{2}=\left[\begin{array}{cc}
0.5 & 0 \\
0 & 0.5
\end{array}\right], F_{d}=\left[\begin{array}{cc}
0.02 & 0 \\
0 & 0.01
\end{array}\right] .
\end{aligned}
$$

In the simulations below, the unknown perturbation is taken as $\delta(p)=\sin (2 p)$. This function is for illustrative purposes only and any $\delta(p)$ that satisfies $\delta^{T}(p) \delta(p) \preceq I, \forall p \in$ $[0, \alpha-1]$ can be considered.

$=$ The reference trajectory is, see Figure 1(a), taken as

$$
y_{r}(p)=\left\{\begin{array}{cc}
0.75 p, & p \leq 40 \\
30, & 40<p \leq 100 \\
70-0.4 p, & 100<p \leq 150 \\
10, & 150<p \leq 180 \\
0.5 p-80, & 180<p \leq 200 \\
20, & 200<p \leq 240 \\
125-0.4375 p, & 240<p \leq 280 \\
2.5, & 280<p \leq 300
\end{array}\right.
$$

Also, it is assumed that the initial state for each trial is $0, \forall k \geq 1$. Frequency spectrum inspection of the reference signal, see Figure 1(b), shows that the significant harmonics are in the range 0 to $4 \mathrm{~Hz}$, and this is taken as the low frequency range. Hence 
Figure 1. The reference trajectory and its frequency spectrum

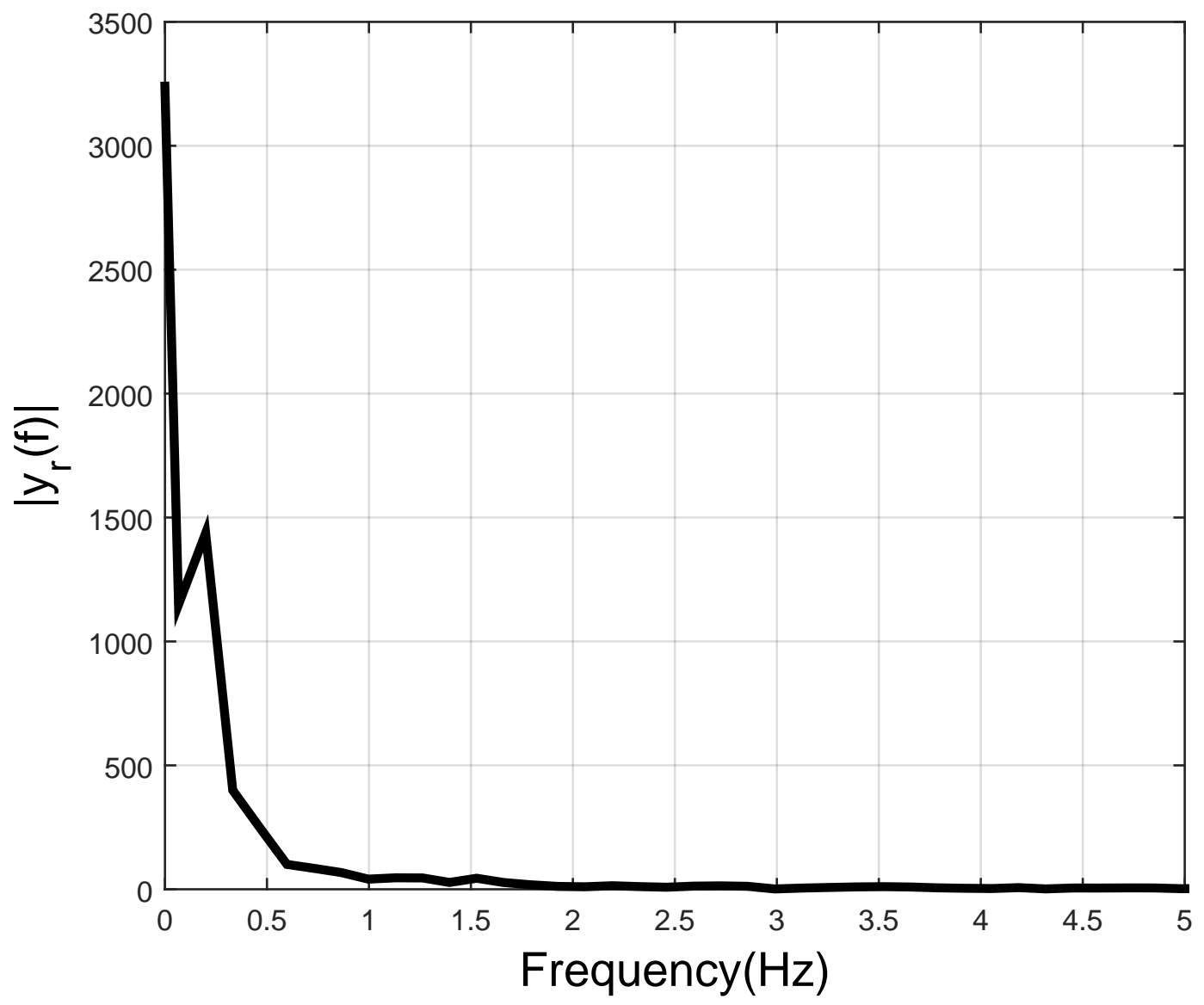


Figure 2. The output tracking error for scenario 1

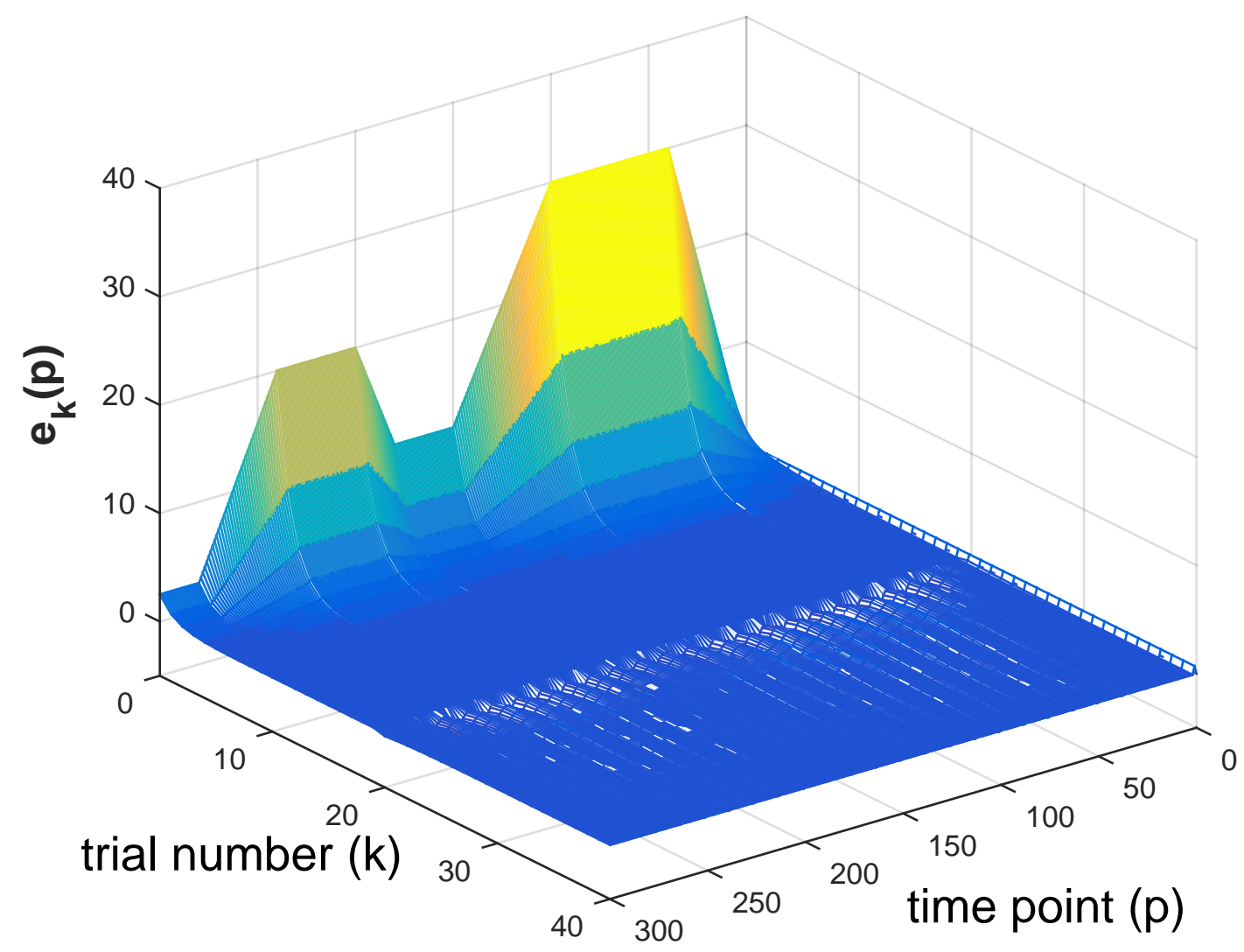


$\theta_{1}=8 \pi \mathrm{rad} / \mathrm{sec}$. Due to the possibility of partial degradation and wear for repeated control operations on some future trial, it is assumed that an unknown actuator fault occurs suddenly after 20th trial in the low frequency range. Also it is assumed that $0.4 \leq \underline{\kappa} \leq \kappa \leq \bar{\kappa}=1$, then $\beta=\operatorname{diag}\{0.5,0.5\}, \beta_{0}=\operatorname{diag}\{0.66,0.66\}$. Hence the time-varying fault factor in simulation is selected as $\kappa=0.7+0.3 \sin (0.5 p)$ and to evaluate the tracking performance from trial-to-trial, the root mean square (RMS) is used, i.e.,

$$
\operatorname{RMS}\left(e_{k}(p)\right)=\sqrt{\frac{1}{301} \sum_{p=0}^{300} e_{k}^{2}(p)} .
$$

Next, two possible scenarios are considered for the maximum possible unit delay.

\section{Scenario 1. Nominal system with a constant fault}

Assume there is no uncertainty in system model matrices. Then applying Theorem 6.1, i.e., solving the LMI (33) for the low frequency interval from 0 to $8 \pi \mathrm{rad} / \mathrm{sec}$, gives

$$
K_{1}=\left[\begin{array}{ll}
-2.0580 & 0.7790
\end{array}\right], K_{2}=0.1562
$$

The simulation results for this design now given. In Figure 2(a), before the fault occurs, the output tracks the desired trajectory with monotonic trial-to-trial error convergence. Once the fault occurs, the output responses deviate from the reference trajectory and the tracking performance deteriorates from trial 20. However, the tracking performance recovers as the trials proceed and eventually recovers the fault-free performance. This is more clearly visible in Figure 2(b) and Figure 3(a), which show that the error will increase when the fault occurs and quickly converge under the fault tolerant control input in Figure 3(b).

\section{Scenario 2. Uncertain system with a time-varying fault}

Consider the case when uncertainty is present and represented by the same $\delta(p)$ as in Scenario 1. Then application of Theorem 6.2 gives the control law matrices

$$
K_{1}=\left[\begin{array}{ll}
-1.8631 & 0.7289
\end{array}\right], K_{2}=0.1342
$$

The simulation results for this case are now given, where the trial-to-trial convergence rate of the error is significantly decreased but acceptable control is still obtained. This example is for a two state SISO problem but, in general, the only limitation relates to solving the required LMIs. No difficulties in this respect are likely for a wide range of possible application areas.

\section{Conclusions}

This paper has developed new results on the iterative learning fault tolerant tracking control problem for a class of linear discrete time-delay systems with actuator faults. A robust fault tolerant ILC law based on the repetitive process theory and GKYP lemma has been developed. Sufficient conditions for the existence of a monotonically 
Figure 3. Control performance for scenario 1

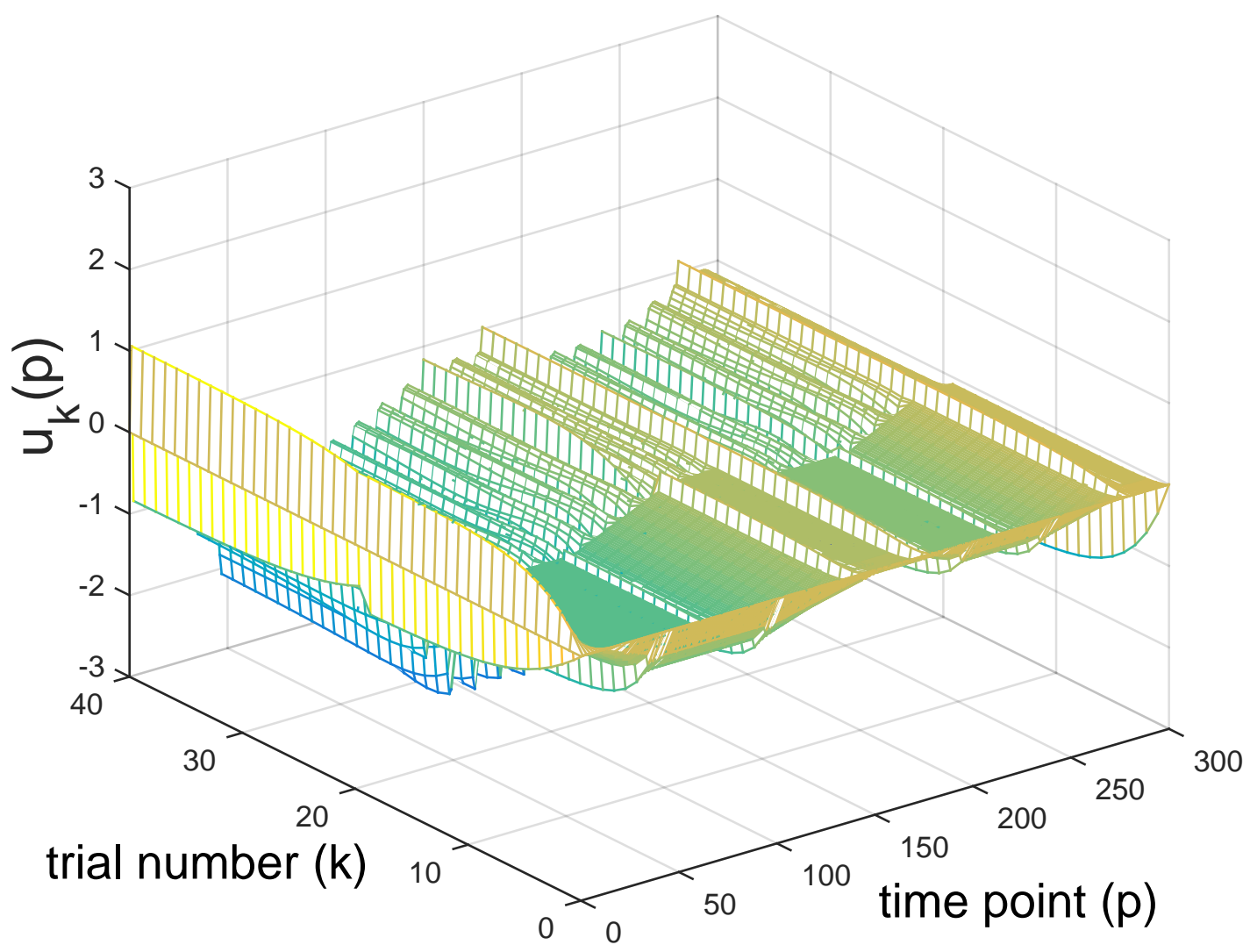


Figure 4. The output tracking error for scenario 2

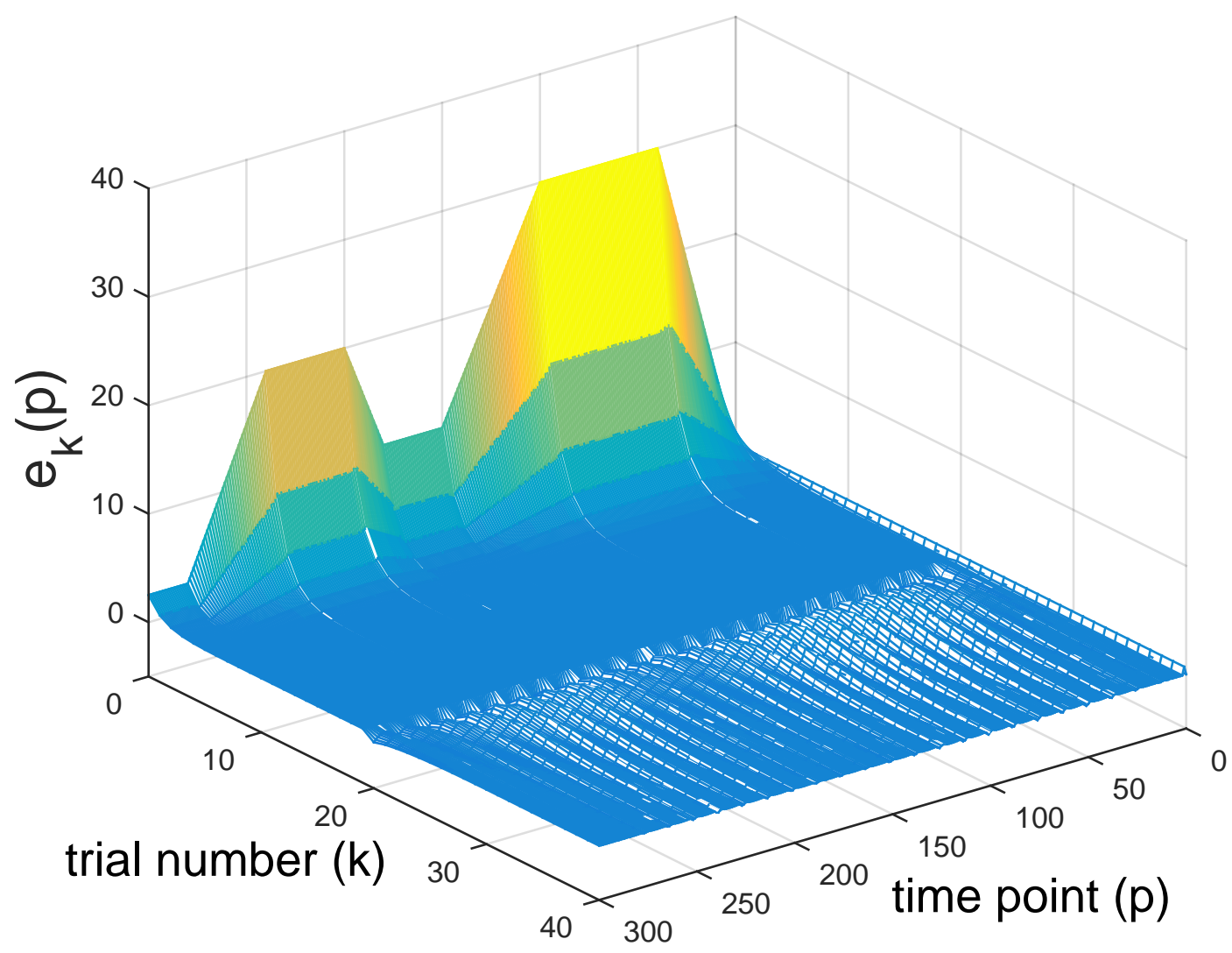


Figure 5. Control performance for scenario 2

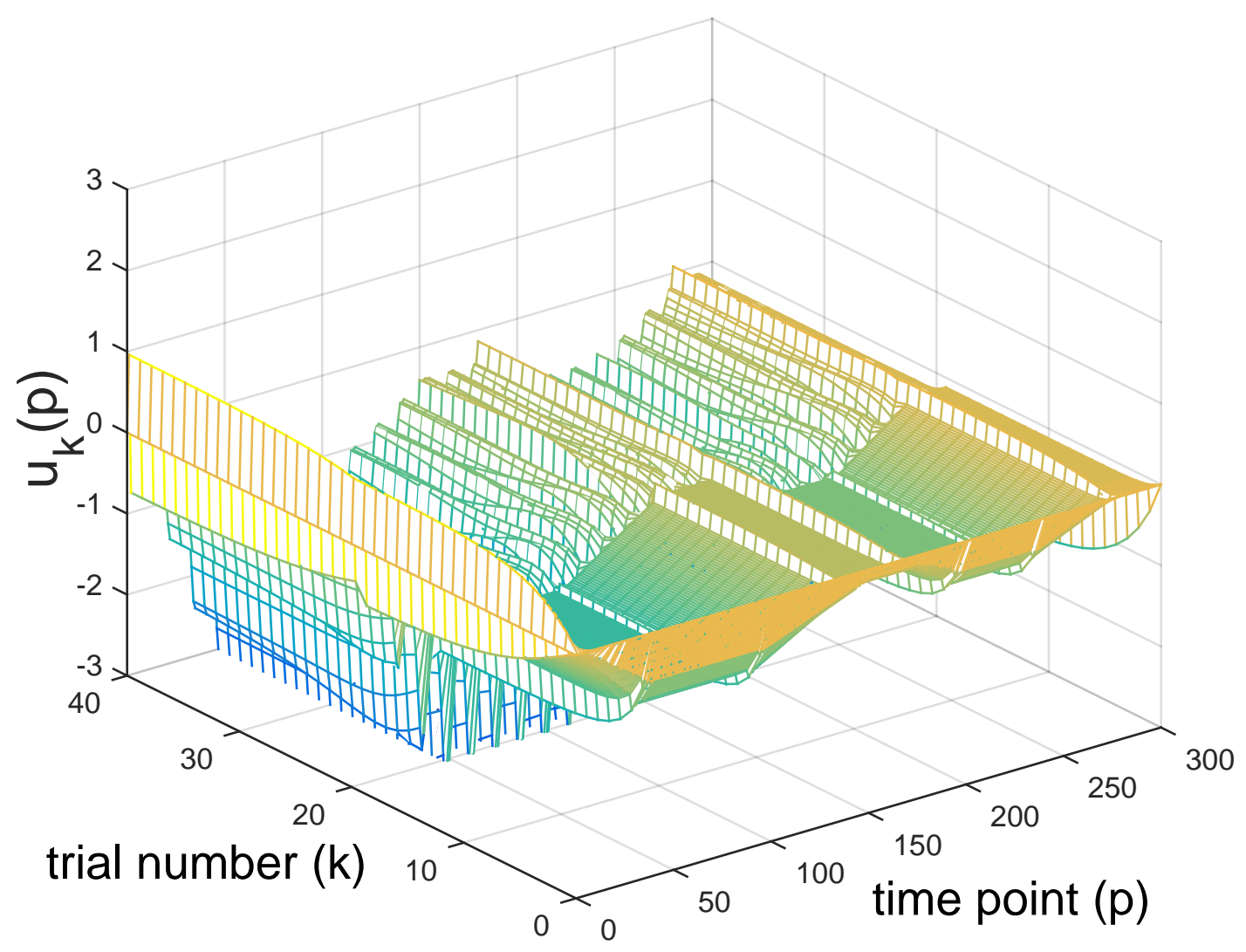


convergent ILC law have been obtained in LMI form and thereby allowing practically relevant control requirements over restricted frequency ranges to be imposed. A simulation based case study demonstrates the effectiveness of the new design method.

Future research should include a detailed investigation into the $\mathcal{H}_{\infty}$ robust performance in the presence of external bounded non-repetitive disturbances in both the state and output vectors. Another area is design of an ILC law where the more complex controller structures are used, e.g. a dynamic controller. Also the extension to multiple state and input delays should also be investigated, together with the effects of uncertainty in the state initial vector on each trial. Finally, when appropriate, experimental validation should be undertaken. More generally, further development of the links between ILC and the subject area highlighted by Heertjes et al. (2016) should be investigated.

\section{References}

Ahn, H., Chen, Y., \& Moore, K. (2007). Iterative learning control: Brief survey and categorization. IEEE Trans Syst Man Cybern C, 36(6), 1099-1121.

Bristow, D., Tharayil, M., \& Alleyne, A. (2006). A survey of iterative learning control: A learning-based method for high performance tracking control. IEEE Control Systems Magazine, 26(3), 96-114.

Gahinet, P., \& Apkarian, P. (1994). A linear matrix inequality approach to $\mathcal{H}_{\infty}$ control. International Journal of Robust and Nonlinear Conrol, 4 (4), 421-448.

Gu, K., Kharitonov, V., \& Chen, J. (2003).

Heertjes, M., Van der Velden, B., \& Oomen, T. (2016). Constrained iterative feedback tuning for robust control of a wafer stage system. IEEE Transactions on Control Systems Technology, 24(1), 56-66.

Hladowski, L., Galkowski, K., Cai, Z., Rogers, E., Freeman, C., \& Lewin, P. (2010). Experimentally supported $2 \mathrm{~d}$ systems based iterative learning control law design for error convergence and performance. Control Engineering Practice, 18(4), 339-348.

Iwasaki, T., \& Hara, S. (2005). Generalized KYP lemma: unified frequency domain inequalities with design applications. IEEE Transactions on Automatic Control, 50(1), 41-59.

Kurek, J., \& Zaremba, M. (1993). Iterative learning control synthesis based on 2-d system theory. IEEE Transactions on Automatic Control, 38(1), 121-125.

Lim, L., Hoelzle, D., \& Barton, K. (2016). A multi-objective iterative learning control approach for additive manufacturing applications. Control Engineering Practice, 46, 157-165.

Longman, R. (2000). Iterative learning and repetitive control for engineering practice. International Journal of Control, 73(10), 930-954.

Meng, D., \& Moore, K. (2016). Learning to cooperate: Networks of formation agents with switching topologies. Automatica, 64, 278-2093.

Paszke, W., Rogers, E., \& Gałkowski, K. (2016). Experimentally verified generalized KYP lemma iterative learning control design. Control Engineering Poractice, 53, 57-67.

Paszke, W., Rogers, E., Galkowski, K., \& Cai, Z. (2013). Robust finite frequency range iterative learning control design and experimental verification. Control Engineering Practice, 21 (10), $1310-1320$

Pipeleers, G., \& Vandenberghe, L. (2011). Generalized KYP lemma with real data. IEEE Transactions on Automatic Control, 56(12), 2942-2946.

Rogers, E., Gałkowski, K., \& Owens, D. (2007). In Control systems theory and applications for linear repetitive processes (Vol. Vol. 349).

Tao, H., Paszke, W., Rogers, E., Yang, H., \& Gałkowski, K. (2017). Iterative learning faulttolerant control for differential time-delay batch processes in finite frequency domains. Journal of Process Control, 56(C), 112-128.

Wang, L., Mo, S., Qu, H., et al. (2013). $\mathcal{H}_{\infty}$ design of $2 \mathrm{~d}$ controller for batch processes 
with uncertainties and interval time-varying delays. Control Engineering Practice, 21(10), $1321-1333$.

Wang, L., Mo, S., Zhou, D., et al. (2012). Robust delay dependent iterative learning faulttolerant control for batch processes with state-delay and actuator failures. Journal of Process Control, 22(7), 1273-1286.

Wang, L., Mo, S., Zhou, D., et al. (2013). Delay-range-dependent robust 2d iterative learning control for batch processes with state delay and uncertainties. Journal of Process Control, $23(5), 715-730$.

Wang, Y., Gao, F., \& Doyle III, F. (2009). Survey on iterative learning control, repetitive control, and run-to-run control. Journal of Process Control, 19(10), 1589-1600.

Zhang, X., \& Yang, G. (2011). Delay-dependent filtering for discrete-time state-delayed systems with small gain conditions in finite frequency ranges. International Journal of Adaptive Control and Signal Processing, 25(11), 983-1005. 